\title{
APLICAÇÃO DE METODOLOGIAS FTIR DE TRANSMISSÃo E FOTOACÚSTICA À CARACTERIZAÇÃo DE MATERIAIS ALTAMENTE ENERGÉTICOS - PARTE II
}

\author{
Elizabeth da Costa Mattos* e Itamar Viganó \\ Gomes, 50, 12228-904 São José dos Campos - SP \\ Rita de Cássia Lazzarini Dutra e Milton Faria Diniz \\ Divisão de Química, Instituto de Aeronáutica e Espaço, Centro Técnico Aeroespacial \\ Koshun Iha \\ Instituto Tecnológico da Aeronáutica, Centro Técnico Aeroespacial
}

Divisão de Sistemas Bélicos, Instituto de Aeronáutica e Espaço, Centro Técnico Aeroespacial, Praça Marechal do Ar Eduardo

Recebido em 29/3/01; aceito 19/12/01

\begin{abstract}
APPLICATION OF FTIR METHODOLOGIES OF TRANSMISSION AND PHOTOACOUSTIC TO THE CHARACTERIZATION OF HIGHLY ENERGETIC MATERIALS - PART II. Materials obtained during the synthesis of octahydro-1,3,5,7-tetranitro1,3,5,7-tetrazocine (HMX) were characterized by Fourier transform infrared (FTIR) transmission spectroscopy and/or Fourier transform infrared photoacoustic spectroscopy (FTIR-PAS). By these techniques the spectrometric alterations that occurred during the process were observed. The characterized species during the synthesis of HMX were $\alpha$-HMX, $\beta$-HMX, hexahydro- $1,3,5-$ trinitro-1,3,5-triazine (RDX) and HMX/RDX mixtures. The FTIR-PAS was verified to be a promising technique of great usefulness of the characterization of highly energetic materials because it is fast, simple and requires no sample preparation unlike Fourier transform infrared transmission technique (KBr pellet). The FTIR-PAS analysis showed that with small sample quantity is possible to distinguish between the $\alpha$-HMX and $\beta$-HMX and to detect even in a qualitative way different HMX / RDX ratios.
\end{abstract}

Keywords: energetic materials; FTIR-PAS; HMX

\section{INTRODUÇÃO}

A síntese de materiais altamente energéticos constitui uma pesquisa importante, desenvolvida nos laboratórios da ASB-CTA, fazendo parte de modernos programas espaciais brasileiros. A elaboração de métodos para a caracterização destes materiais é imperiosa para assegurar a qualidade dos produtos obtidos. Metodologias de cromatografia líquida (HPLC) ${ }^{1}$ vêm sendo empregadas na ASB para tal fim. Atualmente, em nossos laboratórios (AQI-ASB-CTA) estão sendo desenvolvidos métodos que utilizam diferentes técnicas de infravermelho com transformada de Fourier (FTIR) que, associadas ou não a outros métodos, caracterizam e quantificam os materiais energéticos sintetizados ${ }^{2-4}$.

Inicialmente, a preparação da pastilha de $\mathrm{KBr}$, que não altera o teor de cristalinidade do material ${ }^{5}$, foi utilizada na obtenção dos espectros no infravermelho médio (MIR) e distante (FIR) para a caracterização das formas $\alpha$ e $\beta$ de $\mathrm{HMX}^{2}$. Com metodologia simples e rápida foi possível diferenciar essas formas polimórficas de HMX. A identificação da forma $\alpha$ também foi confirmada por meio de difração de raios-X.

Em outro trabalho ${ }^{3}$, no qual utilizaram-se técnicas FTIR de transmissão (pastilha de $\mathrm{KBr}$ ) e reflexão, tais como microscopia-FTIR, na região MIR, e refletância-difusa (DRIFT) na região do infravermelho próximo (NIR), técnica DRIFT-NIR, foi possível estabelecer bandas analíticas para o desenvolvimento e metodologias com possibilidade de aplicação quantitativa para HMX e RDX, dados que foram aproveitados para gerar outra pesquisa ${ }^{4}$.

Nesse estudo ${ }^{4}$, misturas de HMX/RDX foram preparadas, analisadas por meio de cromatografia líquida (HPLC) e por espectroscopia

\footnotetext{
*e-mail: mattos@directnet.com.br
}

no infravermelho de transmissão, nas regiões MIR e NIR. Foi observado que para aplicação dos métodos MIR e NIR é utilizado um número menor de etapas do que a metodologia HPLC, portanto os métodos IR aplicados são mais rápidos. Em adição, foi possível mostrar que os valores encontrados pelas três metodologias, HPLC, MIR e NIR, foram bastante concordantes, e que o método NIR é mais preciso para esse tipo de determinação.

Outras metodologias FTIR, menos convencionais, tais como espectroscopia fotoacústica (PAS) ${ }^{6}$ podem ser aplicadas à caracterização de diferentes materiais. São usadas, em certos casos, em auxílio às técnicas usuais de transmissão, visando avaliação mais completa do material.

A técnica PAS utiliza detecção do sinal acústico de uma amostra gerada por absorção de radiação modulada. A amostra é colocada numa pequena câmara, a qual um microfone é acoplado ${ }^{7,8}$. Radiação modulada é focalizada sobre a amostra, e certas radiações que correspondem ao espectro de absorção do material são absorvidas.

A radiação absorvida causa flutuações de temperatura na superfície. Estas flutuações de temperatura na superfície da amostra induzem a mudanças periódicas de pressão do gás na célula fotoacústica. Uma onda sonora se desenvolve e é detectada por um microfone. Se uma frequiência particular não é absorvida, então, a amostra não aquecerá e nenhuma onda sonora se desenvolverá. Na espectroscopia PAS, ondas sonoras são usadas para detectar freqüências de absorção no infravermelho.

A técnica PAS, de acordo com dados da literatura ${ }^{7,8}$ e experiência em desenvolvimento de metodologias, reúne várias vantagens com relação às técnicas de transmissão: a primeira, é a capacidade de analisar materiais opacos, explorada em compósitos, fibras de carbono, etc.

Com relação à técnica pastilha de $\mathrm{KBr}$, por exemplo, PAS não inclui a interferência de bandas de umidade de $\mathrm{KBr}$, e embora a ex- 
clusão dessa umidade não seja uma tarefa difícil, há necessidade de deixar o material em estufa, entre 100 a $105^{\circ} \mathrm{C}$, além de diminuir o tempo de trituração, pois, como o produto é higroscópico, absorve umidade durante essa etapa, necessária à confecção da pastilha. A eliminação dessas etapas, secagem de $\mathrm{KBr}$ e trituração, constitui um apoio ao uso da técnica PAS. Deve-se registrar que, a utilização da trituração com $\mathrm{KBr}$ na análise do material energético usado nesse trabalho, somente teve o objetivo de evidenciar melhor as bandas, porém o espectro sem a utilização de $\mathrm{KBr}$ é perfeitamente identificável.

Diferentemente dos filmes vazados, técnica onde a amostra deve ser dissolvida em solvente adequado, e o solvente evaporado, em temperatura próxima ao seu ponto de ebulição, de modo que seja formado um filme, PAS não inclui as possíveis interações do material com solvente, alterações de cristalinidade e destruição da orientação molecular. Essas duas últimas são também consideradas na utilização da técnica "melt" (filme prensado a quente).

Com relação às técnicas de reflexão, ATR, DRIFT e microscopiaFTIR, desde que na técnica PAS não há contribuição de radiações refletidas e espalhadas para o aquecimento da amostra, anomalias causadas por esse fenômeno podem ser evitadas.

DRIFT e ATR envolvem, respectivamente, redução de tamanho de partículas e diluição de $\mathrm{KBr}$ em certos casos, e bom contato ótico entre o material analisado e o cristal utilizado para a análise. A geração de sinal da espectroscopia PAS é processada automaticamente e isola, de modo reprodutível, uma camada existente abaixo da superfície da amostra.

Finalmente a espectroscopia PAS no infravermelho pode ser aplicada para amostras em que outras técnicas IR falharem para fornecer espectro de qualidade?.

De todas estas considerações, ficou claro que o pesquisador deve estar atento à escolha adequada da técnica FTIR de análise, que ditará a informação que pode ser obtida de um espectro. A escolha da melhor técnica dependerá do propósito do estudo ${ }^{10}$.

Os resultados encontrados pelos autores em trabalhos anteriores ${ }^{2-4}$, que mostraram-se adequados à caracterização de materiais altamente energéticos sintetizados no CTA, associados às vantagens oferecidas pela técnica PAS no infravermelho em relação às técnicas de transmissão, estimularam a continuidade do desenvolvimento de novas metodologias FTIR para caracterizar amplamente esses materiais.

Este novo trabalho, envolvendo somente métodos FTIR (parte II) visa caracterizar, por meio de técnicas de transmissão, os materiais de partida de uma síntese de HMX e seus intermediários, bem como, as formas $\alpha$ e $\beta$ de HMX por meio de espectroscopia PAS no infravermelho.

Em adição, desde que o Laboratório Instrumental (CIN) da AQI possui equipamento FTIR com ampla faixa espectral (MIR, NIR e FIR) e acessórios tais como microscopia-FTIR, DRIFT, e fotoacústico (PAS), de última geração, espera-se com o desenvolvimento destes métodos estar contribuindo com a comunidade científica, servindo de apoio a outros Centros de Pesquisa e indústrias que utilizam materiais similares. Um exemplo disto é que na literatura consultada não foi encontrada a análise FTIR dos intermediários da síntese de HMX e/ou análise PAS das formas polimórficas deste explosivo e de misturas HMX/RDX.

\section{PARTE EXPERIMENTAL}

\section{Síntese de HMX}

Para síntese de HMX foram utilizadas amostras de partida: hexametilenotetramina (Ultra), paraformaldeído (Synth), e nitrato de amônio (Qeel).
A metodologia empregada para a produção do HMX é baseada no processo de Bachmann, de acordo com o esquema 1(síntese de HMX). No início da reação utiliza-se uma solução de fundo (ácido acético, anidrido acético e paraformaldeído) dando sequiência à reação de nitração da hexametilenotetramina ("hexamina") com solução de ácido nítrico/nitrato de amônio em presença de anidrido acético, responsável pela absorção de água gerada no processo ${ }^{11}$.

Durante o processo de nitração, a temperatura deve ser controlada, pois a reação é altamente exotérmica e a mistura inadvertida dos reagentes de Bachmann pode levar à explosão ${ }^{12}$.

Na primeira etapa deve ocorrer a conversão da hexamina em 2,6 - dinitro (biciclo) pentametilenotetramina (DPT) ${ }^{11}$, que passa por um processo de aquecimento durante alguns minutos denominado de primeira cura, ou seja, tempo necessário para que toda hexamina seja convertida. Numa segunda etapa de nitração onde a solução de ácido nítrico/nitrato de amônio e anidrido acético são dosados, ocorre a formação do HMX, com pequena formação de RDX. Terminada esta etapa inicia-se a segunda cura, ou seja, tempo necessário para garantir a reação completa de nitração, onde são eliminados os produtos instáveis gerados nas etapas anteriores.

Após o final da segunda cura, inicia-se a hidrólise e em seguida a temperatura é elevada para $98{ }^{\circ} \mathrm{C}$ onde os produtos instáveis são decompostos e o anidrido acético em excesso convertido em ácido acético.

$\mathrm{O}$ material aquoso é resfriado a $30^{\circ} \mathrm{C}$ e descarregado em filtro a vácuo. A parte sólida é lavada com água quente, o suficiente para eliminar a acidez do produto. O produto sintetizado é uma mistura rica em HMX com pequenas porcentagens de RDX, e que é separado na fase final do processo; esse subproduto será referido como BMX por se tratar de uma mistura de RDX com baixas porcentagens de HMX.

As amostras utilizadas neste trabalho foram sintetizadas de acordo com metodologia descrita anteriormente ${ }^{2}$, conforme o esquema 1, apenas retirando-se alíquotas, devidamente codificadas, imediatamente após a nitração ou em tempos determinados das etapas de cura, da seguinte forma:

- SU 15/00 - primeira e segunda nitração, onde as amostras foram retiradas imediatamente após cada etapa de nitração;

- SU 16/00 - após a primeira nitração retirou-se uma amostra decorridos 10 min da etapa denominada primeira cura, e após o término da segunda nitração uma amostra decorridos 20 min da segunda cura;

- SU 17/00 - após a primeira nitração retirou-se uma amostra decorridos 20 min da etapa denominada primeira cura, e uma amostra decorridos 90 min da segunda cura.

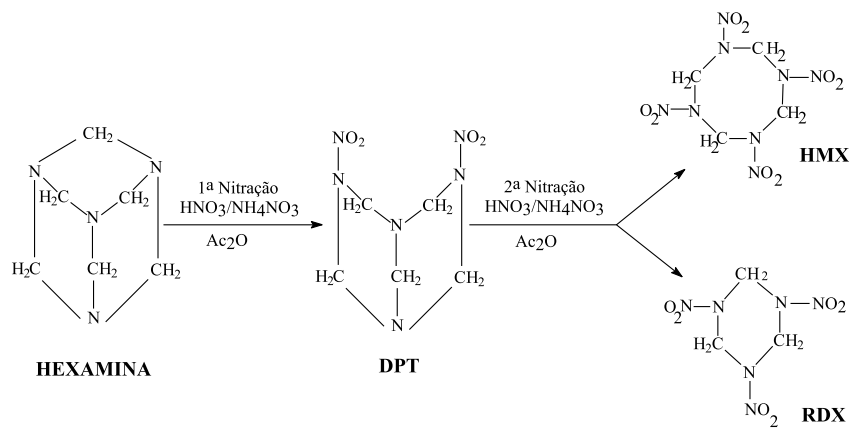

Esquema 1. Sintese de $H M X$

Amostras de HMX (síntese 11/99), BMX SU 14/99 (8,18\% de HMX e 91,82\% de RDX), BMX SU 10/99 (16,04\% de HMX/ 83,96 de RDX), BMX SU 16/99 (28,21\% de HMX/ 71,79\%de RDX), $\alpha$ 
HMX $<65$ \#, foram sintetizadas como descrito anteriormente ${ }^{2}$. A amostra de $\alpha \mathrm{HMX}<65$ \# foi obtida por recristalização do material em solução de $\mathrm{HNO}_{3}$ a $70 \%$, seguida de uma classificação granulométrica ${ }^{13}$, usando-se uma série de peneira tyler, de modo a obter cristais maiores.

\section{Caracterização de HMX por FTIR}

Os espectros FTIR foram obtidos com o espectrômetro FTIR SPECTRUM 2000 PERKIN-ELMER. Os espectros MIR de transmissão foram obtidos com resolução $4 \mathrm{~cm}^{-1}$, ganho 1 , região espectral 4000 a $400 \mathrm{~cm}^{-1}$ e 40 varreduras. Os espectros PAS foram obtidos com acessório PAS MTEC modelo 300, nas seguintes condições: purga com gás hélio, região espectral 4000 a $400 \mathrm{~cm}^{-1}$, resolução 4 , ganho 7 no amplificador, velocidade $0,05 \mathrm{~cm} / \mathrm{s}, 32$ varreduras.

As amostras de HMX, seus materiais de partida e intermediários de síntese, foram preparadas para obtenção do espectro MIR de transmissão ${ }^{14}$ como pastilhas de $\mathrm{KBr}$ (1: $400 \mathrm{mg}$ ), assim como as misturas de HMX/RDX obtidas na síntese de $\operatorname{HMX}(0,8: 400 \mathrm{mg})$.

Para os espectros PAS, as amostras foram diluídas em $\mathrm{KBr}$, em proporção 1:20 mg KBr, maceradas em gral de ágata e analisadas, tendo como espectro de referência o negro de fumo compactado (padrão MTEC) .

\section{Caracterização de RDX por FTIR}

O espectro IR da amostra de RDX, referência cromatográfica (Mantiqueira), foi obtido na forma de pastilha de $\mathrm{KBr}(1: 400 \mathrm{mg})$.

\section{RESULTADOS E DISCUSSÃO}

\section{Análise por FTIR dos produtos de partida de HMX}

Neste item serão posicionadas as bandas características dos materiais de partida de HMX, visando a sua utilização para o acompanhamento das alterações espectrométricas FTIR (aparecimento, desaparecimento, aumento, diminuição, deslocamento de bandas) encontradas ao longo do processo. Os espectros dos materiais de partida, $\alpha$ e $\beta$ HMX obtidos na ASB e de referência, encontrados na literatura $^{5}$, estão incluídos na Figura 1 . A Tabela 1 inclui a atribuição dos grupos funcionais de $\alpha$ e $\beta$ HMX aos modos vibracionais IR correspondentes.

Algumas abreviações desses modos são utilizadas: $v_{\mathrm{a}} \mathrm{e} v_{\mathrm{s}}$ (modos de deformação axial ou estiramento, a-assimétrico, s-simétrico), $\delta_{\mathrm{a}}, \delta_{\mathrm{s}}, \gamma$ e $\tau$ (modos de deformação angular).

A comparação com o espectro de referência, pela similaridade encontrada, permite que o espectro do $\alpha$ HMX, obtido em nossos laboratórios, seja utilizado como referência para o estudo das alíquotas retiradas durante a síntese. As principais absorções observadas nos espectros, associadas a uma provável atribuição ${ }^{14-18}$ e discutidas em cada item, correspondem às características de cada produto.

Um cuidado deve ser tomado, no entanto, na interpretação de espectros obtidos por pastilha, desde que existem bandas em $\sim 3400$ $\mathrm{cm}^{-1} \mathrm{e} \sim 1640 \mathrm{~cm}^{-1}$, associadas à umidade de $\mathrm{KBr}$, que podem interferir na atribuição de bandas características das amostras.

\section{Análise por FTIR de hexametilenotetramina (hexamina)}

A amostra de hexamina (Ultra) foi analisada por FTIR e apresentou bandas em $2921 \mathrm{~cm}^{-1}\left(\mathrm{v}_{\mathrm{a}} \mathrm{CH}_{2}\right), 1457 \mathrm{~cm}^{-1}\left(\delta \mathrm{CH}_{2}\right), 1238 \mathrm{~cm}^{-1}$ ( $v \mathrm{CN}$ ), 811 e $672 \mathrm{~cm}^{-1}$ (vibração do anel) e que correspondem às mesmas bandas observadas para o espectro de referência do composto $^{19}$ encontrado na literatura.

\section{Análise por FTIR de nitrato de amônio}

A amostra de nitrato de amônio (QEEL) foi analisada por FTIR e apresentou as bandas $3129 \mathrm{~cm}^{-1}\left(\mathrm{v} \mathrm{NH}_{4}^{+}\right), 1384$ e $825 \mathrm{~cm}^{-1}$ (vibração do ânion $\mathrm{NO}_{3}^{-}$) que podem ser utilizadas para caracterizar o composto $^{15}$.

\section{Análise por FTIR de paraformaldeído}

As absorções em $2923 \mathrm{~cm}^{-1}\left(v_{\mathrm{a}} \mathrm{CH}_{2}\right), 1469 \mathrm{~cm}^{-1}\left(\delta_{\mathrm{s}} \mathrm{CH}_{2}\right), 1097$, 933 e $905 \mathrm{~cm}^{-1}$ ( $\vee \mathrm{CO}$ éter alifático) confirmam as características encontradas no espectro de referência do composto na literatura ${ }^{16}$, ou seja, revelam que a unidade estrutural $\mathrm{CH}_{2} \mathrm{O}$ está presente.
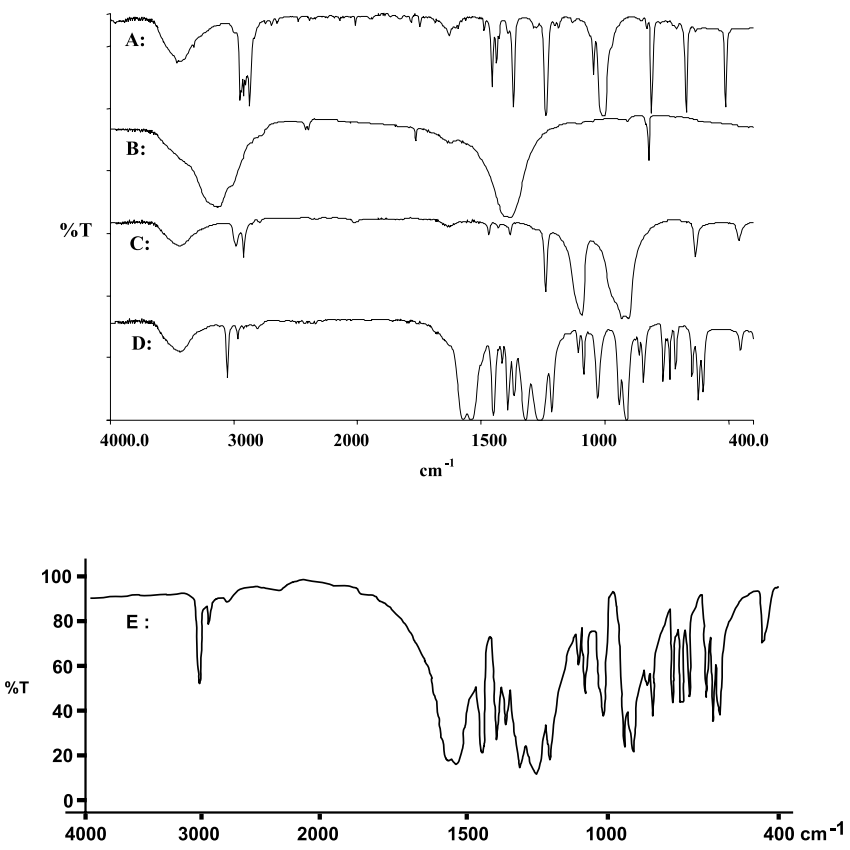

Figura 1. Espectros FTIR de Transmissão: A) hexamina (Ultra); B) nitrato de amônio (Qeel); C) paraformaldeído Synth; D) a HMX (15/00); E) $\alpha$ HMX (referência- literatura $\left.{ }^{5}\right)$

\section{Análise por FTIR de HMX síntese 11/99}

As principais bandas observadas no espectro FTIR do HMX síntese11/99, associadas a uma provável atribuição $0^{5,14,19,20}$, estão na Tabela 1 e Figura 2. O conjunto de bandas observado indica basicamente a presença da forma $\beta$ HMX encontrada na literatura ${ }^{5,14}$.

\section{Análise por FTIR de RDX}

As principais absorções observadas no espectro FTIR do RDX referência cromatográfica, associadas a uma provável atribuição ${ }^{5,14,21}$, estão incluídas na Tabela 1 e Figura 3. As bandas observadas são características de RDX, de acordo com a literatura ${ }^{21}$.

Análise por FTIR de alíquotas retiradas durante o processo de síntese de HMX associada à análise do produto final

As principais absorções para as alíquotas, associadas a uma provável atribuição ${ }^{14,16,18,22}$, estão em números de onda que caracterizam a presença de diferentes compostos, como discutidos a seguir, sugerindo que houve a reação esperada, ou seja a síntese de HMX. 

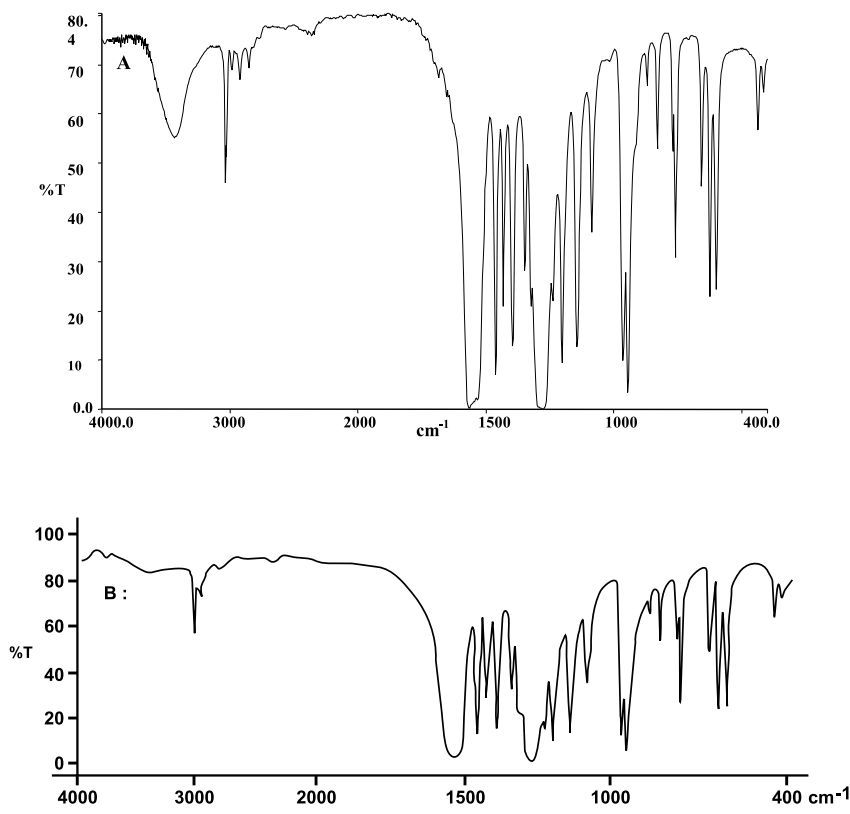

Figura 2. Espectros FTIR de transmissão: A) $\beta$ HMX (SU 11/99); B) $\beta$ HMX (referência- literatura ${ }^{5}$ )

Tabela 1 . Atribuição provável dos grupos funcionais de $\beta \mathrm{HMX}$ e RDX aos modos vibracionais IR correspondentes ${ }^{18}$

\begin{tabular}{|c|c|c|c|}
\hline \multicolumn{2}{|c|}{$\begin{array}{l}\text { NÚMERO DE } \\
\text { ONDA }\left(\mathrm{cm}^{-1}\right)\end{array}$} & \multirow{2}{*}{$\begin{array}{l}\text { NÚMERO DE } \\
\text { ONDA }\left(\mathrm{cm}^{-1}\right) \\
\text { RDX }\end{array}$} & \multirow[t]{2}{*}{$\begin{array}{l}\text { MODO VIBRACIONAL/ } \\
\text { GRUPO FUNCIONAL }\end{array}$} \\
\hline$\beta$ HMX & $\alpha \mathrm{HMX}$ & & \\
\hline 3035 & 3053 & 3073 & $v \mathrm{CH}_{2}$ \\
\hline 1564 & 1572 & 1592 & $v_{\mathrm{a}} \mathrm{NO}_{2}$ \\
\hline 1462 & 1448 & 1459 & $\delta_{\mathrm{s}}^{\mathrm{a}} \mathrm{CH}_{2}^{2}$ \\
\hline 1432 & & 1433 & \\
\hline 1396 & 1384 & 1390 & $\delta_{\mathrm{s}} \mathrm{CH}_{2}$ \\
\hline 1347 & & 1351 & $\delta_{s}^{s} \mathrm{CH}_{2}^{2}$ \\
\hline 1279 & 1277 & 1270 & $v_{\mathrm{s}} \mathrm{NO}_{2}+v \mathrm{~N}-\mathrm{N}$ \\
\hline 1202 & 1213 & & \\
\hline 1145 & 1109 & & $v \mathrm{~N}-\mathrm{N}+v$ anel \\
\hline \multirow[t]{2}{*}{1087} & 1087 & & \\
\hline & 1016 & & \\
\hline 964 & 913 & 1039 & Vibração do anel \\
\hline 946 & & 945 & \\
\hline 830 & 841 & & $\delta$ e $\gamma\left(\mathrm{NO}_{2}\right)$ \\
\hline \multirow[t]{2}{*}{761} & 765 & & \\
\hline & 734 & & \\
\hline 625 & 645 & 604 & $\tau+\gamma\left(\mathrm{NO}_{2}\right)$ \\
\hline \multirow[t]{2}{*}{600} & 616 & & \\
\hline & 606 & & \\
\hline
\end{tabular}

Na comparação de espectros, deve-se levar em conta a interferência, já citada, das bandas de umidade de $\operatorname{KBr}\left(\sim 3400\right.$ e $\left.1640 \mathrm{~cm}^{-1}\right)$, que é dependente do tempo de trituração, necessária à preparação da amostra para a análise IR.

\section{Análise por FTIR de SU 15/00 - primeira nitração}

Bandas em 3050 e $3002 \mathrm{~cm}^{-1}\left(v \mathrm{CH}\right.$ ou $\left.v \mathrm{CH}_{2}\right), 2930 \mathrm{~cm}^{-1}\left(v \mathrm{CH}_{2}\right)$, $1676 \mathrm{~cm}^{-1}(v-\mathrm{O}-\mathrm{N}=\mathrm{O}$ trans $) 1456 \mathrm{~cm}^{-1}\left(\delta_{\mathrm{s}} \mathrm{CH}_{2}\right), 1437 \mathrm{~cm}^{-1}\left(\delta \mathrm{CH}_{2}\right.$ cíclico), $1398 \mathrm{~cm}^{-1}\left(\delta_{\mathrm{s}} \mathrm{CH}_{2}\right), 1384 \mathrm{~cm}^{-1}\left(\delta_{\mathrm{s}} \mathrm{CH}_{3}\right.$ ou v $\left.\mathrm{NO}_{2}\right), 1274 \mathrm{~cm}^{-1}$
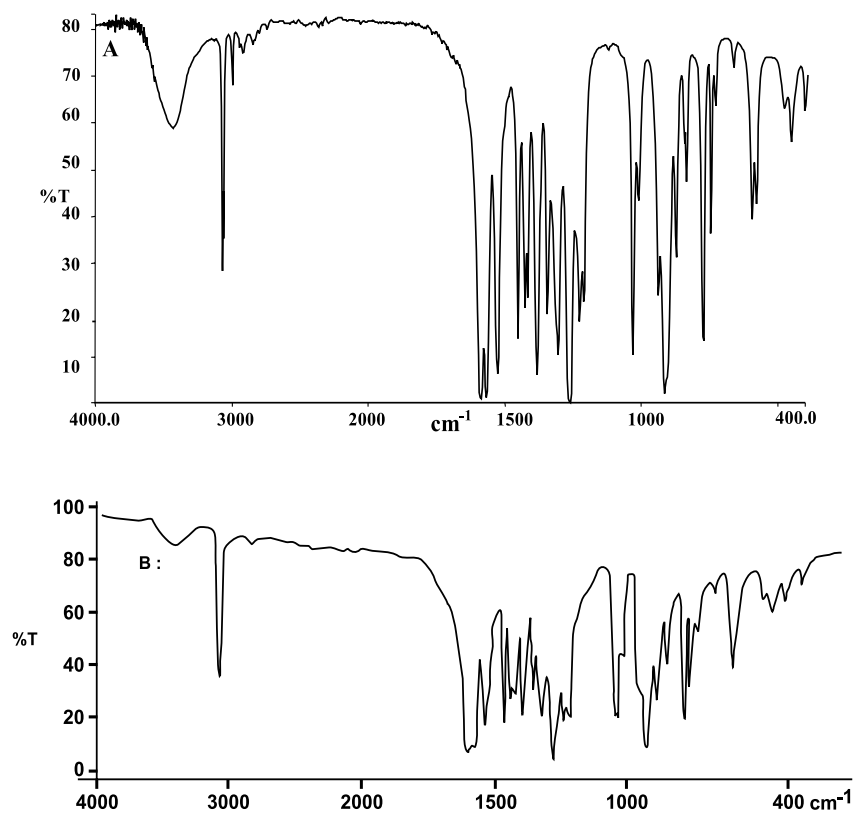

Figura 3. Espectro FTIR de transmissão de RDX: A) RDX mantiqueira; B) $R D X$ (referência da literatura) ${ }^{21}$

$\left(v_{\mathrm{s}} \mathrm{NO}_{2}+v \mathrm{~N}-\mathrm{N}\right), 1205 \mathrm{~cm}^{-1}\left(v \mathrm{NO}_{2}+v \mathrm{~N}-\mathrm{N}\right), 1081 \mathrm{~cm}^{-1}(v$ anel $), 824$ $\mathrm{cm}^{-1}\left(\delta\right.$ e $\left.\gamma \mathrm{NO}_{2}\right), 768 \mathrm{~cm}^{-1}\left(\delta\right.$ e $\left.\gamma \mathrm{NO}_{2}\right), 750 \mathrm{~cm}^{-1}(v \mathrm{NO})$ sugerem que deve ter havido ciclização com um número de $\mathrm{CH}_{2}$ diferente do encontrado na hexamina, pois é observada uma banda relativa a esse grupo em número de onda menor $\left(1437 \mathrm{~cm}^{-1}\right)^{22}$. Além disto, a estrutura deve conter grupos $\mathrm{NO}_{2}$ e, provavelmente, alguma parte alifática.

Esses dados podem estar associados à presença de grupos encontrados na estrutura de composto intermediário DPT. Além disto, estão em concordância com a literatura pois, segundo Urbanski ${ }^{23}$, a síntese de HMX, com a variação de materiais de partida e/ou acidez do meio, pode resultar em misturas ricas de HMX e/ou RDX, além de incluir composto linear (1,9-diacetoxi-2,4,6,8-tetranitro-2,4,6,8tetranonano).

\section{Análise por FTIR de SU 15/00 - segunda nitração}

As principais absorções FTIR da amostra SU 15/00 - segunda nitração, associadas a uma provável atribuição ${ }^{14,18}$, estão em: 3125 e $3053 \mathrm{~cm}^{-1}$ ( $v \mathrm{CH}$ ou $\left.v \mathrm{CH}_{2}\right), 2853 \mathrm{~cm}^{-1}\left(v_{\mathrm{s}} \mathrm{CH}_{2}\right), 1572 \mathrm{~cm}^{-1}\left(\mathrm{v}_{\mathrm{a}} \mathrm{NO}_{2}\right)$, $1448 \mathrm{~cm}^{-1}\left(\delta \mathrm{CH}_{2}\right), 1384 \mathrm{~cm}^{-1}\left(\delta_{\mathrm{s}} \mathrm{CH}_{2}\right.$ ou v $\left.\mathrm{NO}_{\mathrm{s}}\right), 1277$ e $1213 \mathrm{~cm}^{-1}$ $\left(v_{\mathrm{s}} \mathrm{NO}_{2}+v \mathrm{~N}-\mathrm{N}\right), 1109,1087,1016 \mathrm{e} 913 \mathrm{~cm}^{-1}$ ( $v$ anel), 841, $765 \mathrm{e}$ $734 \mathrm{~cm}^{-1}\left(\delta\right.$ e $\left.\gamma \mathrm{NO}_{2}\right), 645,616$ e $606 \mathrm{~cm}^{-1}\left(\tau\right.$ anel e $\left.\gamma \mathrm{NO}_{2}\right)$.

A diferença observada entre os espectros $\mathrm{B}$ e $\mathrm{C}$, na região de 3000 e $3500 \mathrm{~cm}^{-1}$ está associada à presença de grupos $\mathrm{OH}$, referentes à umidade de $\mathrm{KBr}$, e aos grupos $\mathrm{CH}_{2}$ e $\mathrm{CH}$ da alíquota. Entretanto, o conjunto de bandas, principalmente na região de impressão digital (abaixo de $1500 \mathrm{~cm}^{-1}$ ) associado ao seu formato, já sugere a formação ${ }^{18}$ de $\alpha$ HMX, que é confirmada quando se compara os espectros das alíquotas retiradas durante o processo ao espectro do produto final ou seja, do material energético sintetizado HMX SU 15/00 (espectro C) e ao de $\alpha$ HMX (espectro D), Figura 4, já referenciado ao espectro da literatura ${ }^{5}$ (espectro E da Figura 1).

\section{Análise por FTIR de SU 16/00 - primeira nitração após 10 min}

Outro tipo de estudo envolveu a retirada de alíquotas com tempo determinado. As principais absorções FTIR observadas em $2983 \mathrm{~cm}^{-1}\left(v_{\mathrm{a}}\right.$ 


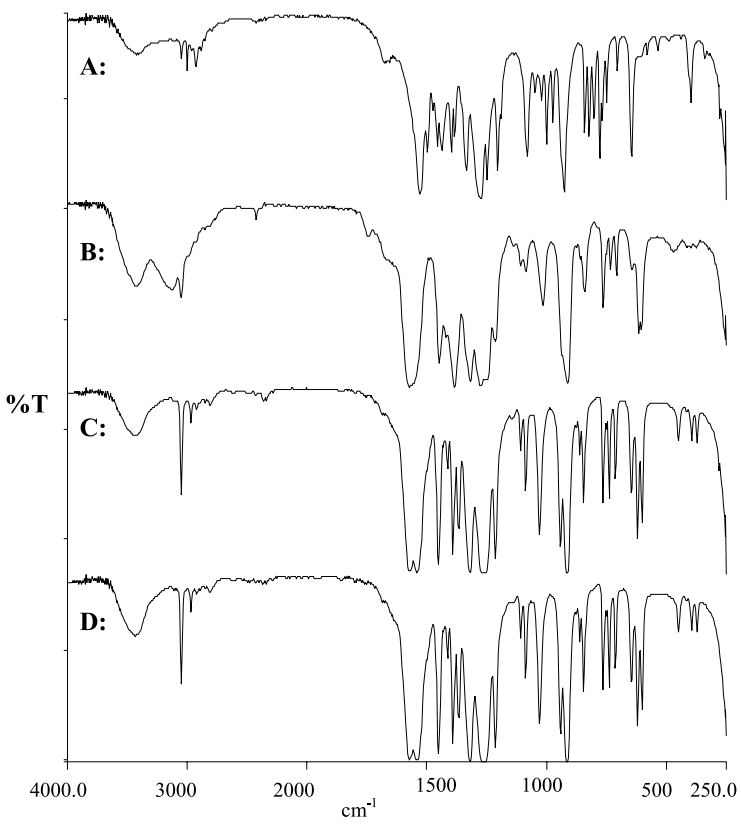

Figura 4. Espectros FTIR de transmissão A) SU 15/00 - 1a nitração; B) SU 15/00 - 2 $2^{\underline{a}}$ nitração; C) $S U$ 15/00; D) $\alpha$ HMX

$\left.\mathrm{CH}_{2} \mathrm{CO}\right), 2923$ e $1469 \mathrm{~cm}^{-1}\left(v_{\mathrm{a}}\right.$ e $\left.\delta_{\mathrm{s}} \mathrm{CH}_{2}\right), 1671 \mathrm{~cm}^{-1}(v-\mathrm{O}-\mathrm{N}=\mathrm{O}$ trans ou $\mathrm{C}=\mathrm{O}), 1275$ e $1206 \mathrm{~cm}^{-1}\left(v_{\mathrm{s}} \mathrm{NO}_{2}+v \mathrm{~N}-\mathrm{N}\right), 1098$ e $906 \mathrm{~cm}^{-1}$ ( $v$ CO éter) sugerem que esta estrutura contenha ainda grupos do paraformaldeído e os nitros, relativos ao processo de nitração (Figura 5).

\section{Análise por FTIR de SU 16/00 - segunda nitração após 20 min}

As principais absorções FTIR observadas para esta alíquota estão em: 3125 e $3052 \mathrm{~cm}^{-1}$ ( $v \mathrm{CH}$ ou $\left.\vee \mathrm{CH}_{2}\right), 1572 \mathrm{~cm}^{-1}\left(\mathrm{v}_{\mathrm{a}} \mathrm{NO}_{2}\right)$, $1449 \mathrm{~cm}^{-1}\left(\delta \mathrm{CH}_{2}\right), 1384 \mathrm{~cm}^{-1}\left(\delta_{\mathrm{s}} \mathrm{CH}_{2}\right.$ ou v $\left.\mathrm{NO}_{2}\right), 1276$ e $1215 \mathrm{~cm}^{-1}$ $\left(v_{\mathrm{s}} \mathrm{NO}_{2}+v \mathrm{~N}-\mathrm{N}\right), 109,1088,1016 \mathrm{e}^{9} 913 \mathrm{~cm}^{-1}$ ( $v$ anel), 845, $765 \mathrm{e}$ $735 \mathrm{~cm}^{-1}\left(\delta\right.$ e $\left.\gamma \mathrm{NO}_{2}\right), 646,617$ e $605 \mathrm{~cm}^{-1}\left(\tau\right.$ anel e $\left.\gamma \mathrm{NO}_{2}\right)$.

Novamente, observa-se uma diferença entre os espectros, nesse caso, (Figura 5) C e D, na região de 3000 e $3500 \mathrm{~cm}^{-1}$, já citado na Figura 4. Porém, as bandas, principalmente na região de impressão digital e seu perfil espectral, sugerem já a formação de $\alpha$ HMX, que é evidenciada quando é feita a comparação com o espectro do material energético sintetizado HMX SU 16/00 (espectro D), Figura 5.

\section{Análise por FTIR de SU 17/00 - primeira nitração após 20 min}

As principais absorções FTIR desta alíquota estão em $3050 \mathrm{e}$ $3002 \mathrm{~cm}^{-1}\left(v \mathrm{CH}\right.$ ou $\left.\mathrm{CH}_{2}\right), 2932 \mathrm{~cm}^{-1}\left(v_{\mathrm{a}} \mathrm{CH}_{2}\right), 1671 \mathrm{~cm}^{-1}(v-\mathrm{O}-\mathrm{N}=\mathrm{O}$ trans), $1456 \mathrm{~cm}^{-1}\left(\delta_{\mathrm{s}} \mathrm{CH}_{2}\right), 1437 \mathrm{~cm}^{-1}\left(\mathrm{v} \mathrm{CH}_{2}\right.$ cíclico $), 1398 \mathrm{~cm}^{-1}\left(\delta_{\mathrm{s}}\right.$ $\left.\mathrm{CH}_{2}\right), 1274$ e $1206 \mathrm{~cm}^{-1}\left(v_{\mathrm{s}} \mathrm{NO}_{2}+v \mathrm{~N}-\mathrm{N}\right), 1080 \mathrm{~cm}^{-1}(v$ anel $)$, $824 \mathrm{~cm}^{-1}\left(\delta\right.$ e $\left.\gamma \mathrm{NO}_{2}\right), 768 \mathrm{~cm}^{-1}\left(\delta\right.$ e $\left.\gamma \mathrm{NO}_{2}\right), 750 \mathrm{~cm}^{-1}(v \mathrm{NO})$.

Este conjunto de absorções sugere a presença de composto cíclico, com número diferente de grupos $\mathrm{CH}_{2}$ do que o encontrado para hexamina, pois há decréscimo no número de onda ${ }^{22}\left(\mathrm{~cm}^{-1}\right)$ de $\mathrm{CH}_{2}, \mathrm{em}$ relação ao espectro desta amina. Da mesma forma que a estrutura da primeira nitração (SU 15/00), além dos grupos $\mathrm{NO}_{2}$ contêm, provavelmente, alguma parte alifática. Estes dados podem estar associados à presença de grupos encontrados em composto intermediário DPT e estrutura alifática, conforme já foi discutido para a amostra SU 15/00.

\section{Análise por FTIR de SU 17/00 - segunda nitração após 90 min}

As principais absorções FTIR desta alíquota estão em 3125 e

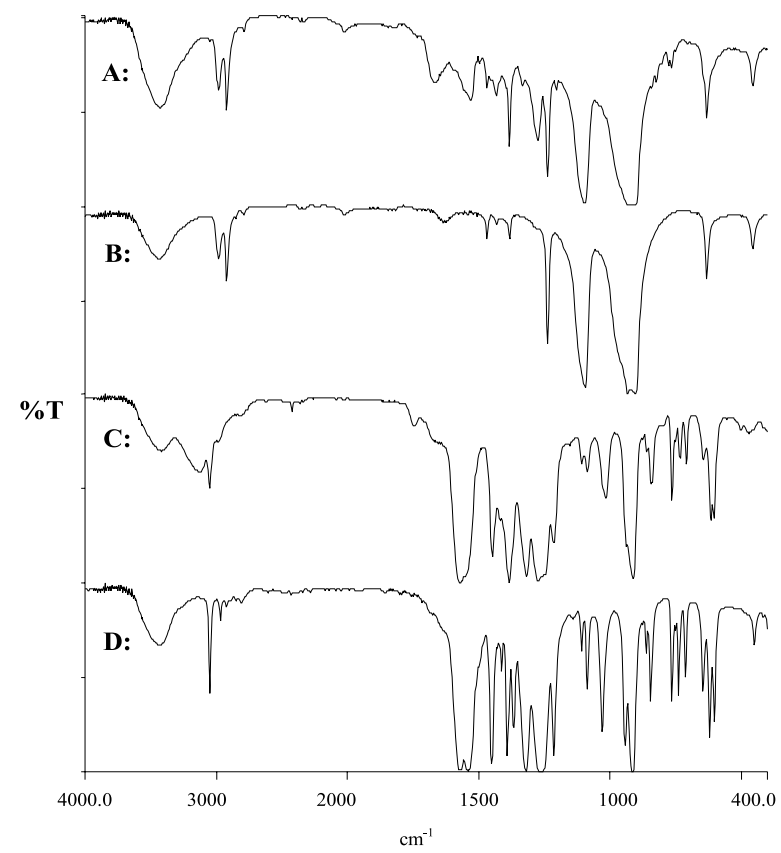

Figura 5. Espectros FTIR de transmissão A) SU 16/00 - 1a nitração; B) paraformaldeído; C) $S U$ 16/00 - 2ª nitração; D) $S U$ 16/00

$3054 \mathrm{~cm}^{-1}\left(v \mathrm{CH}\right.$ ou $\left.v \mathrm{CH}_{2}\right), 1571 \mathrm{~cm}^{-1}\left(v_{\mathrm{a}} \mathrm{NO}_{2}\right), 1449 \mathrm{~cm}^{-1}\left(\delta \mathrm{CH}_{2}\right)$, $1384 \mathrm{~cm}^{-1}\left(\delta_{\mathrm{s}} \mathrm{CH}_{2}\right.$ ou $\left.v_{\mathrm{s}} \mathrm{NO}_{2}\right), 1276$ e $1216 \mathrm{~cm}^{-1}\left(v_{\mathrm{s}} \mathrm{NO}_{2}+v \mathrm{~N}-\mathrm{N}\right)$, 1109,1088 e $914 \mathrm{~cm}^{-1}$ ( $v$ anel), 846, 765 e $^{2} 736 \mathrm{~cm}^{-1}$ ( $\delta$ e $\left.\gamma \mathrm{NO}_{2}\right), 646$, 618 e $604 \mathrm{~cm}^{-1}\left(\tau\right.$ anel e $\left.\gamma \mathrm{NO}_{2}\right)$.

Este conjunto de bandas, associadas ao seu perfil espectral, sugere já a formação ${ }^{18}$ de $\alpha \mathrm{HMX}$, que é confirmada quando se comparam os espectros das alíquotas retiradas durante o processo ao espectro do produto final, o material energético sintetizado HMX SU 17/00 (Figura 6).

Conforme citado (Figuras 4 e 5), a diferença observada entre os espectros B e C (Figura 6), na região de 3000 e $3500 \mathrm{~cm}^{-1}$ está associada à presença de grupos $\mathrm{OH}$, referentes à umidade de $\mathrm{KBr}$, e aos grupos $\mathrm{CH}_{2}$ e $\mathrm{CH}$ da alíquota. Entretanto, a formação do $\alpha \mathrm{HMX}$ é evidenciada comprando-se o espectro (Figura 6B), principalmente na região de impressão digital às absorções do material energético sintetizado HMX SU 17/00, espectro C da Figura 6.

\section{Análise FTIR-PAS de amostras de HMX e HMX/RDX}

Visando obter espectros de explosivos com a mínima preparação de amostra, e compará-los aos obtidos por transmissão IR foi feita a análise FTIR-PAS de HMX e HMX/RDX. Segundo a literatura, a espectroscopia PAS já apresenta bons resultados para sólidos em comparação a outras técnicas FTIR ${ }^{24}$. Essa aplicação da técnica PAS complementa a ampla caracterização FTIR de explosivos, proposta pela nossa pesquisa.

A amostra $\beta$ HMX foi diluída em $\mathrm{KBr}$ apenas para fornecer espectro com bandas mais evidenciadas, sendo então obtido o espectro FTIR-PAS. Suas principais absorções estão em $3033 \mathrm{~cm}^{-1}$ ( $v \mathrm{CH}$ ou $\left.v \mathrm{CH}_{2}\right), 1564$ e $1528 \mathrm{~cm}^{-1}\left(v_{\mathrm{a}} \mathrm{NO}_{2}\right), 1458$ e $1395 \mathrm{~cm}^{-1}\left(\delta \mathrm{CH}_{2}\right), 1292$ e $1200 \mathrm{~cm}^{-1}\left(v_{\mathrm{s}} \mathrm{NO}_{2}+v \mathrm{~N}-\mathrm{N}\right), 1141,1085$ e $945 \mathrm{~cm}^{-1}(v$ anel $), 827 \mathrm{e}$ $761 \mathrm{~cm}^{-1}\left(\delta\right.$ e $\left.\gamma \mathrm{NO}_{2}\right), 658,624$ e $599 \mathrm{~cm}^{-1}\left(\tau\right.$ anel e $\left.\gamma \mathrm{NO}_{2}\right), 436 \mathrm{~cm}^{-1}$ ( $\gamma$ anel), confirmando as bandas assinaladas para a forma $\beta$ de HMX na literatura ${ }^{18}$

A amostra $\alpha$ HMX < 65\#, também diluída em KBr para a obtenção do espectro FTIR-PAS revela como principais absorções as encontradas em $3047 \mathrm{~cm}^{-1}$ ( $\vee \mathrm{CH}$ ou $\left.\vee \mathrm{CH}_{2}\right), 1564 \mathrm{~cm}^{-1} \mathrm{e} 1542 \mathrm{~cm}^{-1}(\mathrm{v}$ $\left.\mathrm{NO}_{2}\right), 1450 \mathrm{~cm}^{-1}\left(\delta \mathrm{CH}_{2}\right), 1321,1292$ e $1218 \mathrm{~cm}^{-1}\left(v_{\mathrm{s}} \mathrm{NO}_{2}+v \mathrm{~N}-\mathrm{N}\right)$, 


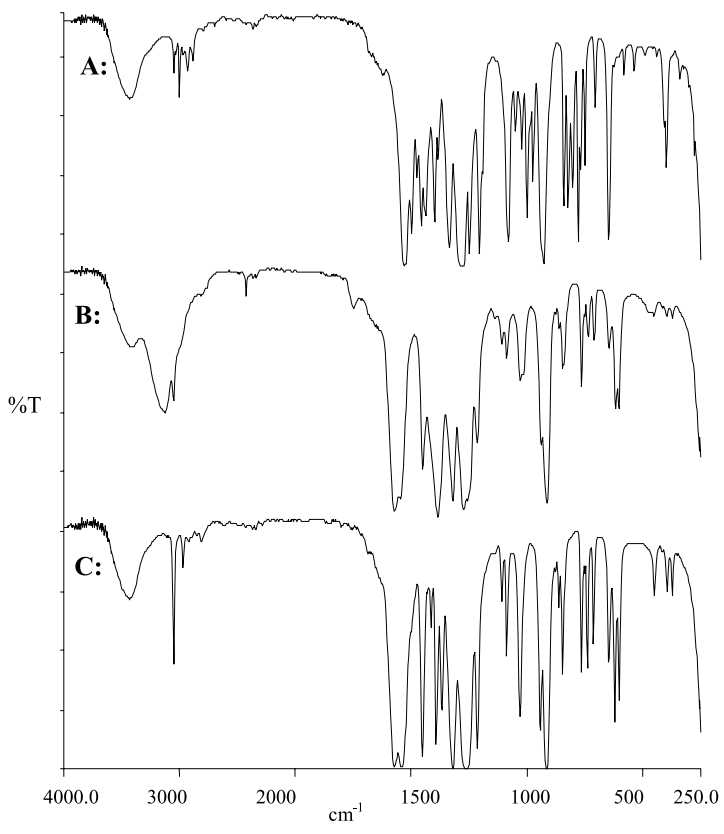

Figura 6. Espectros FTIR de transmissão: A) SU 17/00 - 1ª nitração - após $20 \mathrm{~min}$; B) $S U 17 / 00$ - 2ª nitração após $90 \mathrm{~min} ; \mathrm{C}) \alpha \mathrm{HMX}$

1085, 1030, 941 e $912 \mathrm{~cm}^{-1}$ ( $v$ anel), 846 e $764 \mathrm{~cm}^{-1}\left(\delta\right.$ e $\left.\gamma \mathrm{NO}_{2}\right), 643$, 621 e $599 \mathrm{~cm}^{-1}\left(\tau\right.$ anel e $\left.\gamma \mathrm{NO}_{2}\right), 451 \mathrm{~cm}^{-1}(\gamma$ anel). Essas bandas confirmam a forma $\alpha$ HMX atribuídas na literatura ${ }^{18}$.

A Figura 7 inclui os espectros FTIR de transmissão e PAS das formas $\alpha$ e $\beta$ de HMX. Pode ser observado que as absorções PAS são similares aos de transmissão (espectros em absorvância), em números de onda característicos dos produtos, já discutidos para espectros obtidos por pastilha de $\mathrm{KBr}$.

A amostra BMX SU 10/99, também diluída em KBr para a obtenção do espectro FTIR-PAS, mostra como principais absorções as encontradas em: $3070 \mathrm{~cm}^{-1}\left(v \mathrm{CH}\right.$ ou $\left.\vee \mathrm{CH}_{2}\right), 1590 \mathrm{~cm}^{-1}\left(v \mathrm{~N}-\mathrm{NO}_{2}\right)$, $1568 \mathrm{~cm}^{-1}\left(v_{\mathrm{a}} \mathrm{NO}_{2}\right), 1458 \mathrm{~cm}^{-1}\left(\delta_{\mathrm{s}} \mathrm{CH}_{2}\right), 1317$ e $1266 \mathrm{~cm}^{-1}\left(v_{\mathrm{s}} \mathrm{NO}_{2}+\right.$ $v$ N-N), 1037, 919 $\mathrm{cm}^{-1}$ ( $v$ anel), $602 \mathrm{~cm}^{-1}\left(\gamma \mathrm{NO}_{2}\right.$ e $\tau$ anel) $458 \mathrm{~cm}^{-1}$ ( $\tau$ anel). Este conjunto de absorções, associado a seu formato, indica a presença de mistura de HMX/RDX, que é melhor evidenciada pela

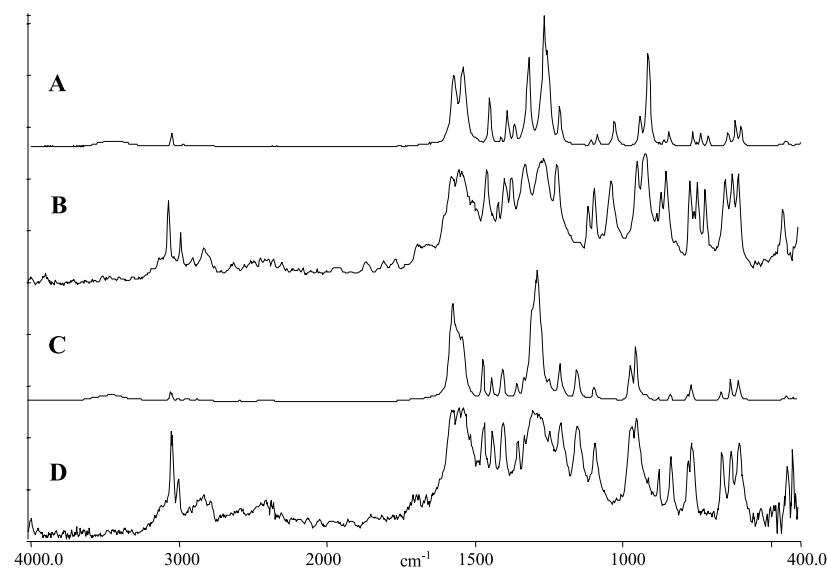

Figura 7. Espectros FTIR de: A) a HMX SU 15/00 - transmissão; B) $\alpha$ HMX SU 15/00 PAS; C) $\beta$ HMX SU 11/99 - transmissão; D) $\beta$ HMX SU 11/ 99 PAS avaliação das diferentes intensidades das absorções características de HMX/RDX nos espectros FTIR-PAS (Figura 8), em 1592 e 1088 $\mathrm{cm}^{-1}$, que correspondem às bandas analíticas utilizadas para estudo quantitativo destas misturas de explosivos por meio de técnicas de transmissão, no trabalho anterior ${ }^{4}$.

Mesmo considerando que a morfologia da superfície da amostra pode afetar a intensidade das absorções ${ }^{25}$ é possível afirmar que há um aumento de intensidade da banda de RDX ou decréscimo da banda de HMX, conforme os diferentes teores destes dois explosivos na mistura mostrando que, mediante uma análise qualitativa FTIRPAS, é possível indicar que está havendo variação dos teores dos explosivos, sugerindo tratar-se de uma técnica em potencial para estudo destes materiais.

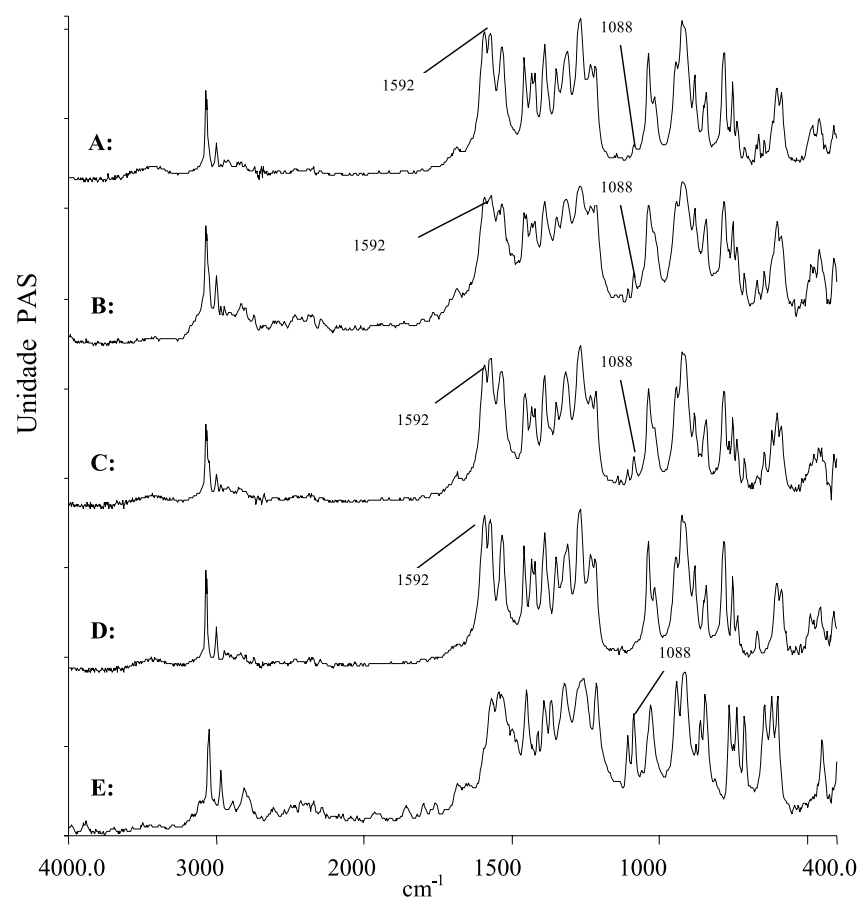

Figura 8. Espectros FTIR-PAS de: A) $S U$ 14/99, B) $S U$ 10/99, C) $S U$ 16/99, D) $R D X, E) \propto H M X$

\section{CONCLUSÃO}

A análise por FTIR de transmissão realizada mostrou que é possível acompanhar as alterações espectrométricas ocorridas durante o processo de síntese de HMX. A análise FTIR-PAS revelou que, com pequena preparação de amostra é possível distinguir $\alpha$ de $\beta \mathrm{HMX}$ e detectar, mesmo que qualitativamente, diferentes teores de HMX/ RDX.

\section{REFERÊNCIAS}

1. Kaiser, M; Propellants Explos. Pyrotech. 1998, 23, 309.

2. Mattos, E. C.; Dutra, R. C. L.; Diniz, M. F.; Otani, C.; Iha, K.; Anais do III Encontro Técnico de Materiais e Química, IpqM, Rio de Janeiro, Brasil, 1999.

3. Mattos, E. C.; Dutra, R. C. L.; Diniz, M. F.; Moreira, E. D.; Iha, K.; Anais do I Encontro Técnico de Química Militar, IpqM, Rio de Janeiro, Brasil, 2000.

4. Mattos, E. C.; Dutra, R. C. L.; Diniz, M. F.; Ribeiro, A. P.; Moreira, E. D.; Iha, K.; trabalho não publicado.

5. Litch, H.H.; $2^{2}$ Symposium on Chemistry Problems with the Stability of Explosives, Tyringe, Sweden, 1970.

6. Yang, C. Q.; Appl. Spectrosc. 1991, 45, 102. 
7. Pandey, G. C.; Kulshreshtha, A .K.; Process Control Qual. 1993, 4, 109.

8. Graf, R. T. ; Koenig, J. L.; Ishida, H.; Polym. Sci. Technol. 1987, $36,1$.

9. Ishida, H.; Rubber Chem. Technol. 1987, 497.

10. Kotova, I. P.; Ivanova, M. P.; Berends, L. K.; Chechetkina, L. N.; Int. Polym. Sci. Technol. 1986, 8, T/66-T/70.

11. Robbins, R; Boswell, B. C. ; US pat. 3,770,721 1973.

12. Leach, J. T.; Staples, J. M.; J. Hazard. Mater. 1981, 4, 271

13. Cady, H. H.; Smith, L. C.; Studies on the polymorphs of HMX, Tech. Rept., $1961 \mathrm{~N}^{\circ}$ : LAMS-2652.

14. Smith, A. L.; Applied Infrared Spectroscopy, John Wiley \& Sons: New York, 1979, p. 286.

15. Miller, F. A.; Wilkins, C. H.; Anal. Chem. 1952, 24, 1253.

16. Pouchert, C.; The Aldrich Lybrary of Infrared Spectra, Aldrich Chemical Company: Wisconsin, 1978, p.115C

17. Wolfgang, W.; Tópicos de Espectroscopia no Infravermelho, Apostila ITA, 1987.
18. Achuthan, C. P.; Jose, C. L.; Propellants Explos. Pyrotech. 1990, 15, 271.

19. Hummel, D. O.; Scholl, F.; Atlas of Polymer and Plastics Analysis, Verlag Chemie GmbH: Weinheim, 1981, vol. 3, p. 6300.

20. Bedard, M.; Heber, H.; Myers, J. L.; Wright, G.; Can. J. Chem. 1962, 46, 2278.

21. Chasan, D. E.; Norwitz, G.; Department of the Army, Philadelphia, 1971, Tech. Rept, T71-6-1.

22. Silverstein, R. M., Bassler, G. C.; Morrill, T. C.; Spectrometric Identification of Organic Compounds, John Wiley \& Sons: New York, 1981, p. 245.

23. Urbanski, T.; Chemistry and Tecnology of Explosives, Pergamon Press: Great Britain, 1984, vol. 4, p. 391.

24. Deeley, C.; Sellors, J.; Spragg, R. A.; Fourier Transf. Spectros. 1989, 1145, 195

25. Pesce-Rodrigues, R. A.; Fifer, R. A.; Appl. Spectrosc. 1991, 45, 417. 